\title{
Participación protagónica de las personas adultas mayores $y$ la actuación profesional del Trabajo Social
}

Recibido: $14 / 04 / 2020$

Aprobado: 27/06/2020

Publicado: 25/08/2020
Jorge García Escobar

Universidad Nacional Mayor de San Marcos

<jgarciae@unmsm.edu.pe>

Joselyn Villa Cavero

Universidad Nacional Mayor de San Marcos $<$ joselyn.villa.cavero@gmail.com>

\section{RESUMEN}

El presente texto tiene como objetivo reflexionar sobre la participación protagónica de las personas adultas mayores y su estrecha relación con la actuación profesional del Trabajo Social. La realidad demográfica del Perú está en constante cambio, generando un fenómeno demográfico progresivo: envejecimiento poblacional. Al mismo tiempo, aún existen estereotipos que encasillan a las y los adultos mayores en un rol de sujeto pasivo. Ante este escenario, ellas y ellos, desde su participación protagónica, y, a través de sus organizaciones de lucha por sus derechos, han fijado las bases para coadyuvar en la construcción de una sociedad inclusiva, que los dignifique como sujetos de derechos y en el marco del paradigma del protagonismo. Frente a esta realidad, el Trabajo Social acompaña, valora y contribuye con la participación activa y decisiva de esta población, visibilizando sus necesidades, demandas y aportes, para luchar con ellas y ellos por una sociedad inclusiva para todas las edades; y, concretando su actuación profesional a través de las políticas públicas.

Palabras Clave: Envejecimiento, personas adultas mayores, participación, protagonismo, Trabajo Social.

\section{Protagonist participation of older adults and professional performance in Social Work}

\begin{abstract}
This text aims to reflect on the protagonist participation of older adults and their close relationship with the professional performance of Social Work. Peru's demographic reality is constantly changing; a progressive demographic phenomenon is developing: population ageing. At the same time, there are still stereotypes that typecast older adults into a passive person role. Faced with this scenario, they since their protagonist participation, and, through their organizations fighting for their rights, have laid the groundwork for contribute to build an inclusive society, that dignify them as subjects of rights and within the framework of the protagonist paradigm. In the face of this reality, Social Work accompanies values and contributes to the active and decisive participation of this population, making visible their needs, demands and contributions, to fight with them for an inclusive society for all ages; and, realizing his professional performance through public policies.
\end{abstract}

KeYword: Aging, older adults, participation, protagonist, Social Work. 


\section{Anotación previa}

L a vejez y el envejecimiento son aspectos actuales de la realidad social. En este marco se destaca la relevancia del cambio demográfico y las concepciones de la sociedad sobre las personas adultas mayores. Asimismo, se reflexiona sobre las posibilidades de participación social de esta población como elemento central en la comprensión de los derechos humanos, y a la vez, como una de las bases de su posicionamiento como sujetos sociales de derechos.

Es decir, mediante el reconocimiento de los derechos humanos y las condiciones de vida de quienes integran/conforman este sector de la población, es posible comprender que no son menos importantes que cualquier otro grupo demográfico y/o ciudadano, a la vez que, ampliamos nuestras perspectivas acerca de las capacidades de las personas adultas mayores como gestoras de sus procesos de desarrollo, encaminados en y desde la promoción y defensa de sus derechos.

Las y los adultos mayores desde el ejercicio de su participación protagónica, por medio de sus organizaciones autónomas, buscan forjar una sociedad inclusiva, cambiando los mitos y estereotipos negativos anclados ${ }^{1}$ en las mentalidades colectivas de nuestras sociedades, y que, se expresan culturalmente como interacciones jerárquicas cotidianas del día a día (Ames, 2011). Por el contrario, esta población organizada busca fomentar un ideario renovado diferencial que los conciba como actores sociales, como sujeto colectivo de derechos capaces y protagonistas de su desarrollo, y sobre todo, en constante lucha por transformar cualquier condición de vulneración de sus derechos y/o exclusión social.

\section{Contexto actual del envejecimiento en el Perú}

Desde aproximadamente la mitad del siglo XX, a nivel mundial, y por correlato en la región de América Latina y el Caribe, viene desarrollándose un fenómeno demográfico progresivo: envejecimiento poblacional, que de acuerdo con lo seńalado por el

1 Tales como representaciones de incapacidad, limitación, inactividad, asexualidad, etc.
Centro Latinoamericano y Caribeño de Demografía (CELADE) está generando diversos impactos, como por ejemplo la desaceleración del bono demográfico ${ }^{2}$ y el incremento poblacional del grupo etáreo (INEI 2020). La población adulta mayor es la de más rápido crecimiento en la sociedad actual (Sánchez, 2005, p. 14). En nuestro país, es el resultado de los grandes cambios demográficos experimentados en las últimas décadas del país. En aquel futuro cercano, se estima para el 2050 que uno de cada cuatro peruanos será una persona adulta mayor.

En los últimos 30 años se han presentado avances políticos y normativos a nivel internacional, latinoamericano, y nacional, hacia un compromiso global a favor del adulto mayor. Desde las Asambleas Mundiales sobre Envejecimiento: la primera realizada en Viena (1982) y la segunda en Madrid (2002), donde se propone el cumplimiento del Plan de Acción Internacional sobre Envejecimiento; y las Conferencias Regionales intergubernamentales sobre envejecimiento en América Latina y el Caribe.

En el Perú, en el año 1999 el Estado designa como ente rector en el tema de adulto mayor al Ministerio de Promoción de la Mujer y del Desarrollo Humano (actualmente Ministerio de la Mujer y Poblaciones Vulnerables - MIMP). En el año 2000, este Ministerio aprueba los lineamientos de política para esta población. Después, se aprueba el Plan Nacional para el Adulto Mayor 2002 al 2006, con activa participación de diversas organizaciones de la sociedad civil; y, se extiende su vigencia hasta el 2010. En el 2006 se establece la Ley de las Personas Adultas Mayores No 28803 que precisa la creación de Centros Integrales de Atención del Adulto Mayor (CIAM) en las Municipalidades provinciales y distritales de todo el Perú. Luego, se aprobaría el Plan Nacional para las Personas Adultas Mayores 20132017. Finalmente, el 21 de julio del 2016, se publica la Ley de la Persona Adulta Mayor - Ley No 30490, y su respectivo reglamento.

Del mismo modo, como país tenemos como pendiente ratificar la Convención Interamericana sobre la Protección de los Derechos Humanos de las Personas Mayores, que fue aprobada el 15 de junio

2 Se entiende la desaceleración del bono demográfico en el sentido del aumento sostenido de la población adulta mayor y crecimiento de la población en edad activa (14 a 59 ańos) frente al decrecimiento sostenido de la población infantil y juvenil. 
del 2015 por la Organización de Estados Americanos (OEA); y, que es de fundamental importancia, toda vez que el mencionado instrumento internacional contribuirá al fortalecimiento de las políticas públicas a favor de las personas adultas mayores de nuestro país.

Es importante tener en cuenta que los instrumentos legales y de políticas públicas trazan las vías de acción estatal en el corto, mediano y largo plazo para la atención y promoción de capacidades de las personas adultas mayores. En ese sentido, estos documentos de gestión pública se han redactado desde una perspectiva de derechos humanos, tratando de posicionar las potencialidades y habilidades del sector social de personas adultas mayores para su participación plena en nuestras sociedades. De esta manera, marcan un punto de transición hacia abordajes y comprensiones más potenciales respecto al sujeto, ello es clave, pues en cada lineamiento normativo y político está presente una representación o paradigma de sujeto de derechos, lo cual es fundamental en tanto posibilitaría aminorar las políticas sociales exclusivamente asistenciales y paternalistas, para más bien tener otras que se enfoquen en el bienestar social e inclusión social integral y acceso a los beneficios del desarrollo de estas personas. Coincidimos plenamente con Remy, cuando plantea que: "en política el paternalismo es la cara publica y comúnmente aceptable del autoritarismo" (Remy, 1991, p. 264).

\section{Evolución histórica de las percepciones y concepciones del adulto mayor}

Desde una mirada histórica y paradigmática, la temática del adulto mayor se ha manifestado mediante ideas variadas e incluso opuestas. Por ejemplo, la conceptualización sobre esta población se ha manifestado y transformado en consonancia con los cambios de la sociedad. Siguiendo esa perspectiva, y de acuerdo a las diferentes etapas históricas de la sociedad peruana, Piña (2009) nos menciona que, en las sociedades primitivas, el anciano ocupaba un lugar primordial, donde la longevidad se vinculaba a la sabiduría y a la experiencia. De modo que, en estas sociedades las personas adultas mayores, tenían una valoración respetable y sobresaliente; siendo considerados porta- dores de conocimiento y representantes de su legado cultural.

En las sociedades preincaicas, según Varela y otros (2004), la estructura de poder teocrática y la base agropecuaria de la producción basaron su supervivencia en dos fuentes: el trabajo de los jóvenes y adultos, y en los conocimientos y saberes de las personas adultas mayores, ocupando estos últimos un lugar importante dentro de la organización social. Durante el incario la valoración positiva de la persona adulta mayor no se vería en términos generales afectada. Al respecto, autores como Quintanar (2000) y Sánchez (2005) refieren la adecuada integración y trato considerado de esta población dentro de las comunidades incas, participando en diversos aspectos relevantes, e inclusive formando parte de consejos de ancianos que tenían un cargo importante en cuanto a temas de salud y educación.

Del mismo modo, Albertin analizando la obra del cronista Pedro Cieza de León plantea la impronta de la opinión de las personas adultas mayores al momento de determinar si un inca había desplegado una gestión optima o deficiente, en sus términos:

Cuando moría [...] se la hacia una gran fiesta y se lloraba por mucho tiempo, sobre todo si había reinado con amor y justicia hacia los súbditos. Si los ancianos determinaban que [...] había sido justo y loable de ser recordado por el futuro, se llamaba a los contadores del reino para que se perpetuase la memoria del difunto. (Albertin, 2012, p. 349)

Sin embargo, después de la invasión española, se instaura una nueva estructura económica y social, generando una fragmentación de nuestra sociedad que da surgimiento de dos versiones de Perú: una república de españoles y una república de indios ${ }^{3}$. En adelante, la perspectiva sobre el adulto mayor tendrá dos versiones: una occidentalizada, criolla, que lo remite al grupo de los no aptos, siendo desvalorizados y omitidos. Y la otra versión, expuesta en las sociedades

3 Ames retomando el parecer de Flores Galindo, opta por denominar al Perú indígena y su devenir en el tiempo como una república sin ciudadanos (Ames, 2011, p. 229). Sobre el origen de esta partición y dualidad de dos Estados que legalmente son solo uno, pero en la práctica son dos, Thurner menciona: "la clave de la solución hispana a este problema fue la invención jurídica, efectuada en el tardío siglo XVI, de la colonizada nación yndica y su república de indios, yuxtapuesta en forma binaria a la nación espańola colonial y su república de espańoles" (Thurner, 2006, p. 31). 
tradicionales y sus descendientes, donde es parte activa de la familia ampliada, reconocido en su aporte y participación en diversas tareas del hogar.

Posteriormente, ocurren cambios al inicio de la etapa Republicana, debido a la influencia de las transformaciones a nivel mundial con la industrialización y los adelantos científicos, se acentúan las redefiniciones en las condiciones de vida de las personas mayores, en zonas urbanas se empieza a resaltar más aspectos negativos de esta población, destacando las deficiencias físicas de las personas adultas mayores y reduciendo, en el ámbito laboral, su capacidad de aporte. En este periodo se acentúa la situación de marginación, se les continúo considerando una fuerza de trabajo desvalorizada, con escasas oportunidades en el mercado laboral.

No obstante, según Pińa (2013), con los nuevos aportes de la medicina, se separan los conceptos de vejez y enfermedad en la tercera edad, existiendo una gran preocupación científica sobre el tema, dando lugar al nacimiento de la gerontología o disciplina del estudio y fomento de capacidades de la persona adulta mayor. Sin embargo, desde algunos sectores de la academia y los campos de intervención social, las personas adultas mayores continúan siendo considerados como los carentes y pobres, bajo un enfoque de objeto de derechos, con el propósito de satisfacer principalmente sus necesidades básicas.

Como bien menciona Miralles (2011), la imagen que la sociedad tiene de las personas adultas mayores, de la vejez como fenómeno social y del envejecimiento como proceso y estado demográfico, está asociada de forma casi automática a elementos negativos. Culturalmente, desde el frente occidental y moderno ha prevalecido una visión de la vejez que tiende a identificar a estas personas adultas como un grupo poblacional supuestamente homogéneo caracterizado por la inactividad, improductividad y dependencia.

Se pierde perspectiva de diversidad al enfocar a este grupo poblacional, seguimos pensando en razón a la unicidad del modelo de sujeto adulto mayor en correspondencia con la presentación colectiva o modelo cultural urbano que centra en un sujeto pletórico de necesidades, sin tener en cuenta que vivimos en sociedades fragmentadas donde la normatividad se disuelve, donde la predictibilidad del comportamiento del sujeto se esfuma (Golte y León, 2011, p. 48), y donde tenemos que buscar comprender desde la diversidad social y la diversidad propia o interna de la persona, que responde a un contexto igual de fragmentado y a una trayectoria de vida no exenta de crisis y contradicciones. Algo que sin duda no ha sido nada fácil para los analistas sociales.

\section{Algunos fundamentos teóricos sobre vejez y envejecimiento}

En el intento de definir la vejez a lo largo del tiempo se han presentado diferentes posturas, una de ellas es de Pérez Ortiz (citado en Aranibar, 2001), quien establece dos dimensiones para conceptualizar la vejez, siendo estas: la edad y la estructura o sistema social. La primera entendida como la gran variable estratificadora u ordenadora que permite comprender la vejez desde los ciclos de vida de las personas; y la segunda, referida a la sociedad y sus reglas que imponen pautas de comportamiento y de conducta, creando la vejez como construcción social.

Además, Aranibar (2001) hace referencia al ciclo de vida, prestando atención a la situación y la posición social experimentada en la vejez, que a su vez se explica por los acontecimientos, decisiones y conductas de las personas en etapas anteriores de la vida, contextualizadas por aspectos sociales e históricos.

En consecuencia, la vejez es una etapa más del proceso del ciclo vital. En ese sentido, se comprende que la vejez no es la única etapa del ciclo de vida que presenta cambios biológicos y fisiológicos en la persona. En otras etapas de vida como la niñez, la adolescencia y la juventud también existen cambios. En otras palabras, las personas adultas mayores pueden experimentar, al igual que en otras etapas, una vida con un total estado de bienestar físico, psicológico y social; y estar expuestos, al igual que los demás, a riesgos o amenazas que puedan influir en su despliegue humano integral.

En la actualidad, el término de envejecimiento activo, tiene la finalidad de retomar la importancia de tener acceso a la salud, haciendo referencia al bienestar físico, mental y social durante esta etapa de vida, esto les permite su participación demostrando sus potencialidades, acompañado de la protección y accesibilidad, que contribuyan al desarrollo en todos los ámbitos acorde a sus intereses y necesidades. 
Estos aspectos conllevarán a obtener una óptima forma de vida.

$\mathrm{Al}$ respecto, Giró indica que:

El envejecimiento activo se aplica tanto a los individuos como a los grupos de población, permitiendo a las personas realizar su potencial de bienestar físico, social y mental a lo largo de todo su ciclo vital. Para lograr una cultura de envejecimiento activo es necesaria la interacción de factores relacionados tanto con el entorno físico como en lo social; factores relacionados con los sistemas sanitarios y sociales; factores económicos y factores de índole personal y de estilos de vida. (Giró, 2011, pp. 27-28)

En lo antes señalado, observamos que Giró conceptualiza el envejecimiento activo en el plano individual y colectivo, pero tiene limitaciones al comprender a la persona como sujeto receptor de políticas de protección, centradas principalmente en su bienestar físico, relacionado por extensión con los aspectos sociales y económicos, omitiendo otras dimensiones de su mundo vital (por ejemplo, vínculos afectivos, salud mental, actividad ocupacional, etc.).

A su vez, la Asociación para el Aprendizaje Permanente y la Participación Social de las Personas Mayores (Hartu-Emanak, 2007) define el envejecimiento activo como:

Proceso que se desarrolla a lo largo de la vida, integrado por políticas, actitudes y actividades, que hacen que dicho envejecimiento sea saludable para la persona que lo vive y rentable para la sociedad en la que vive, para lo cual la persona recibe de la sociedad conforme a sus necesidades y da a la sociedad, según sus capacidades. (Hartu-Emanak, 2007, p. 21).

El envejecimiento activo no es exclusivo de una determinada etapa de vida, se desarrolla en todas las personas a lo largo de su ciclo vital, donde conjugan las necesidades de la persona, las capacidades y potencialidades que posee y los satisfactores de su entorno. No obstante, resulta necesario superar aquel criterio que prioriza solo las necesidades de las personas, y que, bajo esa lupa, considera a las personas adultas mayores como sujetos pasivos y carenciados.

Desde las políticas de envejecimiento activo se visualiza el interés por potenciar las capacidades de la persona al envejecer, de llevar una vida productiva en la sociedad. Sin embargo, ser adultos mayores activos no solo refiere a los ámbitos físico o económico, sino a los otros aspectos de la vida en sociedad, tales como la participación integral en los asuntos sociales, culturales, políticos y otros temas de su interés; es decir, en todos los aspectos que le conciernen para el desarrollo amplio de sus capacidades y potencialidades, así como para el pleno goce de sus derechos.

El Instituto de Mayores y Servicios Sociales (2001), plantea aportes integradores a la propuesta de envejecimiento activo, señalando lo siguiente:

\begin{abstract}
Maximizar el potencial del envejecimiento activo requiere de una estrategia comprensiva que impulse la participación y el bienestar en todas las etapas del curso vital. Envejecer bien es cosa de todos a todas las edades. Supone calidad de vida, participación e incremento de ciudadanía. (Instituto de Mayores y Servicios Sociales, 2011, p. 81)
\end{abstract}

En definitiva, al hablar de envejecimiento activo, nos referimos a todo el proceso vital de la persona, sin establecer un periodo exclusivo para el envejecimiento, sin discriminación por edad y/o generación; en consecuencia, es una categoría/noción inclusiva porque expresa un compromiso de toda la sociedad, para generar condiciones favorables y reconocer a los adultos mayores como personas integrantes de nuestra sociedad. Desde esa perspectiva, la sociedad debe contemplar a todas y todos los ciudadanos por igual, brindándoles las mismas posibilidades en las áreas de educación, protección, derechos y con la implementación de la solidaridad intergeneracional, que a su vez permita sostenerse y salvaguardarse entre grupos poblacionales.

\section{Participación social}

En 1992 Gyarmati (citado por Instituto de Mayores y Servicios Sociales, 2008), define participación como la capacidad real y efectiva del individuo o de un grupo, de tomar decisiones sobre aspectos que directa o indirectamente afectan sus actividades dentro de la sociedad. Por otro lado, Iturrieta (2008), define participación social como un proceso más amplio, 
relacionada a una acción colectiva, colaborativa, de involucramiento por parte de un grupo de individuos que aúnan voluntades a fin de conseguir generar demandas sociales que se expresen en beneficios para la población.

En ese sentido, se entiende por participación a las iniciativas sociales, donde las personas, sujetos o actores sociales, con orientaciones e intereses comunes, en un tiempo y espacio definido, toman decisiones e influyen en los aspectos de su vida social, política, económica y cultural. Hay que mencionar, además, según el Instituto de Estudios Latinoamericanos (s.f.), la participación social es comprendida como una posibilidad de configurar nuevos espacios sociales y/o la inclusión de actores sociales en los movimientos sociales, en organizaciones gubernamentales y no gubernamentales; así como, su incidencia en la cuestión social para denunciar las exclusiones, demandar cambios sustanciales y aportar en la construcción de una sociedad inclusiva.

Se representan aspectos diferentes en las conceptualizaciones de participación. Esta puede ser considerada como medio para el ejercicio de la ciudadanía, dado que es accesible y visible, posibilitando a la persona ejercer sus diferentes capacidades como integrante de un colectivo, para lograr un objetivo de interés común; donde la democracia juega un papel importante.

Alineada en esta perspectiva, el Instituto de Mayores y Servicios Sociales de España (2008) manifiesta que la participación desde las asociaciones ayuda a mejorar la calidad de vida de las personas. Pero, la participación también es una exigencia que nace de la convivencia humana, un compromiso de solidaridad y reciprocidad que enriquece a la persona adulta mayor y fortalece y regenera a la sociedad.

Asimismo, para Hartu-Emanak (2009), la participación de las personas adultas mayores es considerada como una necesidad, como un derecho y como una obligación de todas y todos; y por tanto desarrollada en relación a sus necesidades, capacidades y oportunidades. Igualmente, como un proceso que nace en las bases (desde abajo hacia arriba), proceso cíclico y ascendente, siendo el resultado del movimiento social, forjado en las organizaciones de las personas adultas mayores y del tejido social que ellas mismas, de manera organizada, fueron creando.

\section{Paradigma de protagonismo}

Protagonismo es un concepto en evolución, por ello es necesario un recuento histórico para comprender su desarrollo del concepto y posicionamiento.

Cussiánovich (2010) menciona que, en el contexto de los ańos 60 y 70, los movimientos populares cumplieron un rol importante en las acciones de impacto político, dando origen a la expresión de protagonismo popular. Desde esa perspectiva, el protagonismo se reconoce en las organizaciones sociales de base, en los sindicatos, movimientos sociales de estudiantes, mujeres, jóvenes, entre otros, ocupando un lugar significativo en lo social, en lo político, en escenarios locales, regionales, nacionales y latinoamericanos.

Sin embargo, en las últimas décadas el concepto de protagonismo ha tomado mayor presencia en el plano de la ciudadanía al lograr mayor visibilidad social en las organizaciones sociales en sus diversos escenarios. Según Cussiánovich (2007), el protagonismo se presenta en primer lugar como una necesidad implacable de la especie humana y de cada individuo, y por ello mismo, como un derecho inherente a la condición humana y de las comunidades. De tal forma que, en cierto sector de lo social y político, el protagonismo tomó camino como parte de un sentido común de la gente.

En esa perspectiva, el protagonismo principalmente evoca una afirmación de la persona como actor social, que se agrupa en diversos colectivos en su lucha permanente por transformar cualquier condición de exclusión, manifestándose como sujetos de derechos capaces y protagonistas de su desarrollo.

De acuerdo con Jaramillo (2005), no se nace protagonista espontáneamente, sino que se trata de una vocación a desplegar, por tanto, el protagonismo debe ser asumido y ejercido, en el día a día, en la cotidianidad de la vida de cada persona. Es preciso tomar en consideración que las nociones de protagonismo son producto de una construcción social que se enriquece continuamente por las experiencias de los actores sociales, sus organizaciones y sus comunidades.

En el paradigma del protagonismo, según Alfageme, Cantos y Martínez (2003), la participación implica un salto cualitativo dado que se reviste de exigencias que afectan al conjunto del proceso de ejercicio de su derecho a la actoría social. Por tal razón, el protagonismo no es un simple atributo que se 
agrega a la participación, sino que coloca a las personas, como es el caso de las personas adultas mayores, como actores sociales y no como simples consentidores de las acciones de los ejecutores de las políticas públicas.

En concordancia con lo anterior, el Instituto de Mayores y Servicios Sociales de España (2008) indica que una de las claves para que las personas adultas mayores sigan siendo protagonistas de sus vidas, de prolongar su autonomía e independencia, es tomando conciencia de sus capacidades incluso en los casos de dependencia. Está claro que, nadie sabe mejor que ellos y ellas de sus necesidades, requerimientos y demandas. Por tanto, para que obtengan su plena inclusión en la sociedad, es fundamental que ellos y ellas sean los actores sociales en la toma de decisiones, en la planificación y ejecución de acciones para mejorar su calidad de vida.

Esta idea se refuerza con lo planteado por Bazán (2009) al seńalar que el protagonismo se encarna o toma cuerpo en el emerger de las organizaciones, cuya aparición coloca a la persona como actor y autor, como sujeto social y político. Además, sostiene que el protagonismo es el punto de unión entre la sociedad y el Estado, el punto que anuda el tejido social. En consecuencia, al fusionarse "participación" y "protagonismo" y orientarse hacia su materialización con personas adultas mayores, tenemos que este se refiere a un estilo de participación, siendo sus características principales el auto reconocimiento e identidad como persona adulta mayor, como miembro de un colectivo, portador de solidaridad, humildad, dignidad, respeto, propulsor de protagonismo y con capacidad de actuación y transformación sobre su realidad.

Este nuevo planteamiento implica una profunda revisión de los modelos de participación hasta entonces vigentes, así como un avance hacia enfoques y acciones que posibiliten la inclusión de las voces de las personas adultas mayores en los procesos de decisión a nivel local, regional y nacional. Además, una nueva forma de participación desde un enfoque intergeneracional, que asuma como fin la constitución de una mejor sociedad para todas las edades, donde los actores sociales: personas adultas mayores, sean los y las protagonistas, y tomen las decisiones en las acciones que viabilizan una vida digna.

Sin embargo, lo anterior no quiere decir que este proceso no sea aceptado, apoyado y movilizado por las instituciones, por lo contrario, genera la transformación de un "hacer para" las personas adultas mayores, en "hacer con" las personas adultas mayores, es decir, "nada sobre ellos, sin ellos", o en las voces de las personas adultas mayores: "nada sobre nosotros, sin nosotros".

\section{Acción colectiva y experiencia organizativa de las personas adultas mayores}

En el Perú, las personas adultas mayores están organizados de diversas maneras, motivaciones y razones; entre ellas, organizaciones de pensionistas y/o jubilados, organizaciones religiosas, organizaciones de programas sociales municipales (impulsadas por los Centros Integrales Atención de las Personas Adultas Mayores de los gobiernos locales) y las organizaciones comunitarias. Desde la década de los años 90 hasta la actualidad, sus organizaciones sociales se han incrementado considerablemente; y a la par, la demanda de esta población por el respeto de sus derechos, su participación y capacidad de incidencia política, sobre todo en las organizaciones comunitarias, quienes han puesto en marcha el despliegue de sus capacidades y visibilización de sus potencialidades.

Según Bravo (2011), las organizaciones de personas mayores son un valioso espacio de acompañamiento mutuo, superación; así como, de participación y ejercicio de ciudadanía. En efecto, se trata de organizaciones sociales que se basan principalmente en el compartir de experiencias individuales y colectivas, la defensa de sus derechos humanos, el ejercicio de su ciudadanía y la movilización de recursos. La experiencia se ha venido forjando históricamente y se ha enriquecido con sus experiencias de vida personal y colectiva. Es precisamente la experiencia de su trabajo organizado, lo que ha favorecido el logro de sus demandas; e incluso, una mejor calidad de vida.

Las organizaciones y asociaciones de las personas adultas mayores están representadas y encaminadas por sus mismos protagonistas, es decir, es iniciativa principal de sus integrantes, su participación protagónica se manifiesta en el desarrollo de su actoría social y política. El colectivo de personas adultas mayores tiene una presencia cada vez más relevante en nuestras sociedades y no sólo cuantitativa, sino, 
sobre todo, cualitativa, partiendo desde sus capacidades y potencialidades. Son muchos los valores que pueden aportar como sujetos poseedores de conocimientos y experiencias adquiridas a lo largo de su vida, tienen el derecho de desarrollar una mayor presencia pública y social, adquiriendo el protagonismo que les corresponde en la toma de decisiones, aportando a la sociedad como sujetos colectivos de derechos, y no, como meros espectadores o sujetos pasivos.

\section{Una experiencia de participación protagónica: ADOPAMLO}

Una experiencia destacable es el de la Asociación de Organizaciones de Personas Adultas Mayores del Distrito de Los Olivos ${ }^{4}$ (ADOPAMLO) que se conformó en el año 2007, con un aproximado de 9 organizaciones ${ }^{5}$ a manera de bases, las cuales están agrupadas principalmente por la zona donde residen, seguido por la afinidad e intereses en común de sus integrantes. ADOPAMLO es una red social conformada por organizaciones de personas adultas mayores del distrito de Los Olivos, Lima; y, se encuentra legalmente reconocida en la Superintendencia Nacional de Registros Públicos desde agosto del 2010, lo cual, desde su propia perspectiva, constituye un serio logro en su reconocimiento como sujeto colectivo o personería jurídica.

Las organizaciones que integran ADOPAMLO iniciaron su accionar como espacios de recreación y uso del tiempo libre para personas adultas mayores; sin embargo, en la actualidad buscan tomar un papel importante en el desarrollo de su comunidad y en la sociedad. Es así que, mediante su organización y participación constante, han logrado protagonismo e incidencia en su localidad, mediante las acciones socioeducativas para todas las personas mayores, sensibilización hacia la comunidad para rom-

4 Los Olivos es un distrito de Lima Metropolitana, específicamente ubicado en el Cono Norte. Su población está conformada básicamente por migrantes provenientes de los departamentos del interior del país y su descendencia.

5 Las organizaciones de personas adultas mayores fundadoras de ADOPAMLO, que actualmente se encuentran activas son: "AVAMUPRO", "Parque Naranjal", "Viviendo Felices", "Amigos Unidos", "Edad de Oro", "La Esperanza”, "Carlos Cueto Fernandini" y "Mercurio Alto".

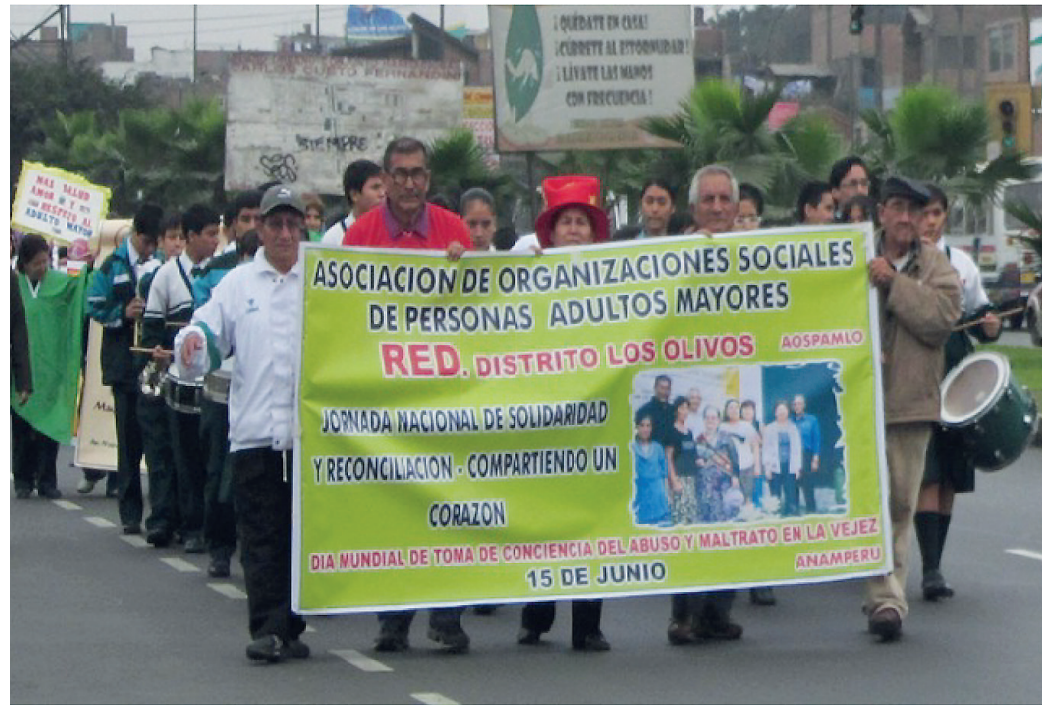

Imagen 1. Asociación de Organizaciones de Personas Adultas Mayores del Distrito de Los Olivos

Fuente: Archivos fotográficos de la Asociación de Organizaciones de Personas Adultas Mayores del Distrito de Los Olivos (ADOPAMLO), 2010.

per estereotipos, defensa de sus derechos humanos, movilización de recursos, articulación con otras organizaciones (de personas adultas mayores de Lima y organizaciones de la sociedad civil que trabajan a favor de las personas mayores) e instituciones públicas (por ejemplo: Centros de Salud, Hospitales, Defensoría del Pueblo, Ministerio de Justicia y Derechos Humanos, Centro Integral de Atención del Adulto Mayor de Los Olivos, entre otras), la demanda y vigilancia constante de la defensa de sus derechos; todo ello, aporta en la revaloración social y política de la persona adulta mayor.

En la actualidad, ADOPAMLO está conformada por 20 organizaciones ${ }^{6}$ de personas adultas mayores, distribuidas a nivel de todo el distrito de Los Olivos, Lima. Para sus integrantes es importante establecer relación con las demás organizaciones, porque les permite crear un espacio de comunicación e interaprendizaje entre personas y agrupaciones. Una integrante activa refiere que:

6 Las organizaciones de personas adultas mayores que actualmente conforman ADOPAMLO son: "AVAMUPRO", "Parque Naranjal", "Viviendo Felices", "Amigos Unidos", "Edad de Oro", "La Esperanza", "Carlos Cueto Fernandini", "Mercurio Alto", "Primavera", "San Conrado", "Renacimiento Olivense", "Río Santa", "Laura Caller", "Flor de Loto", "Inmaculada Concepción”, "La Tercera Edad de la Esperanza", "Virgen de las Nieves", "Seguidores de Cristo", "Las Golondrinas", "Amor y Amistad Campo de Pro". 
totalmente activos (...) es muy importante porque cada ańo van creciendo las instituciones que nos apoyan en esta causa.

Irene Zamudio Luna, integrante de ADOPAMLO (2019).

Un aspecto resaltante en la participación protagónica de los integrantes de ADOPAMLO, es que la gran mayoría de sus integrantes son mujeres. El respecto, el presidente de la Red menciona lo siguiente:

Es una realidad a nivel de todo el Perú, que los adultos mayores varones muy poco participan en las organizaciones de adultos mayores. Aquí debo elogiar a las damas, porque las damas son las que nos han tomado la delantera, y el $90 \%$ son damas las que pertenecen y dirigen las organizaciones de adultos mayores en Los Olivos, en Lima Metropolitana y en el Perú entero, porque he tenido la oportunidad de viajar a Arequipa, Piura, Ica, Huancayo, Chiclayo, donde la mayoría de las organizaciones son manejadas por mujeres.

José Flores Romero, presidente de ADOPAMLO (2019).

La experiencia de ADOMPAMLO es un gran ejemplo de participación protagónica de personas adultas mayores, desde las propias voces de los y las protagonistas, hemos descrito algunas manifestaciones de su involucramiento en el desarrollo social y político. En estos procesos, los y las trabajadores sociales han acompañado a las organizaciones de personas adultas mayores, en el ejercicio y defensa de sus derechos; así como, su incidencia política ante las instituciones del Estado.

\section{Actuación profesional del Trabajo Social en la acción colectiva del adulto mayor}

En el escenario de la temática del adulto mayor, se origina la necesidad de comprender la vejez y el envejecimiento desde una mirada renovada, que favorezca a las personas adultas mayores y en extensión a la sociedad. Dado que, a pesar que existen esfuerzos para retomar la valoración de estas personas, a partir de las Asambleas Mundiales, Latinoamericanas y las acciones peruanas, aún resulta un esfuerzo limitado, puesto que es una tarea pendiente profundizar los debates sobre la problemática del adulto mayor, y materializar en políticas públicas orientadas a la construcción de una sociedad inclusiva para todas las edades, que dignifiquen al adulto mayor como sujeto de derechos, reconociéndolo y visualizando su contribución en la sociedad.

Las y los trabajadores sociales debemos generar marcos interpretativos y procesos investigativos que potencien la teorización y praxis profesional en relación con el envejecimiento y el rol social de las personas adultas mayores, centrándose el interés en el impacto de esta población en la sociedad. Dado que, son actores en el desarrollo de nuestra nación, ciudadanos que ejercen derechos, sujetos sociales que toman decisiones; y no deben ser concebidos como personalidades pasivas que requieren únicamente asistencia y cuidados.

De acuerdo con González (2017), el Trabajo Social en el campo de intervención con las personas adultas mayores, realiza su aporte disciplinario en sus diferentes niveles de abordaje (caso, grupo y comunidad) y desplegando múltiples estrategias tendientes al despliegue de potencialidades y capacidades, así como articulando con actores sociales y redes institucionales y comunitarias, entre otras.

Los colectivos gestados en la participación autónoma e informada de las personas adultas mayores, algunos con una tradición de décadas acumuladas y enfocadas en la promoción y defensa de sus dere$\operatorname{chos}^{7}$, no dejan de ser un llamado de atención e imperativo de acción para las y los trabajadores sociales que buscamos contribuir a la promoción y defensa de los derechos de personas. Estos movimientos expresan el rol de la población adulta mayor que se niega a ser entendida como la sufrida, la plagada de necesidades y la dependiente, por el contrario, estas organizaciones creadas desde la necesidad de espacios de participación y actoría social proponen, exponen, vigilan, conocen sus derechos y el rol político a desempeñar desde la sociedad civil.

La actuación profesional del Trabajo Social debe estar orientada a apoyar y/o acompañar a las coaliciones que van dando forma al gran movimien-

7 Dentro de los diversos modelos de intervención del trabajo social con personas adultas mayores propuestos por Sánchez, consideramos que el procedimiento grupal y más específicamente el de los grupos de acción comunitaria se acerca mejor a una propuesta profesional que fomente el protagonismo social organizado con esta población. 
to social de las personas adultas mayores. A su vez, contribuir en la sensibilización y la formación para la eliminación de los prejuicios y mitos sobre la vejez, proceso de envejecimiento y el rol de las personas adultas mayores en la sociedad, contribuyendo a su visibilización.

Las organizaciones y asociaciones de personas adultas mayores en su diversidad vienen generando estructuras integradoras que buscan en un futuro no lejano configurarse como el gran movimiento social de personas adultas mayores (de diferentes realidades, situaciones y condiciones, zonas de vida, etc.), generando espacios de diálogo en igualdad de oportunidades, creando propuestas colaborativas a ser accionadas colectivamente en la búsqueda de potenciar sus propuestas, agendas sociales de políticas, y niveles de incidencia.

Frente a este escenario actual de la participación de las personas adultas mayores, en relación a los niveles de incidencia real en las políticas públicas del Estado, las y los trabajadores sociales desde nuestra actuación profesional debemos fundamentalmente impulsar la participación protagónica de esta población, enfatizando en la planificación y ejecución de políticas públicas que posicionen a estas personas como actores en el desarrollo de nuestra sociedad peruana, como ciudadanos que ejercen derechos, como sujetos sociales que toman decisiones y jamás deben ser concebidos como sujetos pasivos que requieren únicamente asistencia y cuidado.

\section{Conclusiones}

El actual y futuro contexto peruano refleja una sociedad que progresivamente envejece, pero aun en la sociedad peruana continúan los mitos, estereotipos y percepciones negativas sobre la vejez, el envejecimiento y el aporte de las personas adultas mayores. Frente a este escenario, las propias personas adultas mayores han buscado las sendas para hacer valer y respetar sus derechos, mediante el ejercicio de la participación protagónica.

La participación protagónica implica reconocer a las personas adultas mayores como sujetos sociales de derechos, y, además, nos compromete como sociedad, a facilitar su auto-reconocimiento como ciudadanos, capaces de promover y exigir el cumplimiento de sus derechos, generando propuestas alternativas, participando en la toma de decisiones, ejerciendo control social e incidencia política. El protagonismo de las personas adultas mayores supone una participación libre, voluntaria, democrática y de inclusión social para todas las edades. Las organizaciones y asociaciones de las personas adultas mayores buscan hacer llegar sus necesidades, puntos de vista y propuestas a los Gobiernos Locales y Estado Central a través de su incidencia política y presión social. En su accionar se configuran como una ciudadanía emancipada, libre del control o imposición estatal, en su práctica se van perfilando y definiendo como actores sociales protagónicos.

El compromiso de las y los trabajadores sociales con las personas adultas mayores se debe evidenciar en el fortalecimiento de sus asociaciones y coaliciones con una visión de proyección nacional, la colaboración en la incidencia social y política propuesta por el colectivo de las personas adultas mayores, el acompańamiento en los procesos de establecimiento de agenda social de las organizaciones y la promoción de espacios de diálogo y negociación entre Estado y las organizaciones de estas personas.

\section{Bibliografía}

Albertin, C. (2012). La relación entre el poder incaico y la población en el señorío de los incas de Pedro Cieza de León. En revista Lexis Vol. º36 (2). Lima: Pontificia Universidad Católica.

Alfageme E., Cantos R. y Martínez M. (2003). De la participación al protagonismo, propuestas para la acción. Madrid: Plataforma de Organizaciones de Infancia.

Ames, P. (2011). Cultura y desigualdad. Discriminación, territorio y jerarquías en redefinición. En Cotler, Julio, y Ricardo Cuenca (eds.). Las desigualdades en el Perú: balances críticos. Lima: Instituto de Estudios Peruanos.

Araníbar, P. (2001). Acercamiento conceptual a la situación del adulto mayor en América Latina. Serie Población y Desarrollo Nº. 21. Santiago de Chile: CEPAL/ CELADE.

BAZÁn, J. (2009). El colaborador y el protagonismo de la niñez. Cualidades y descriptores. Lima: Save the Children Canadá.

Bravo, O. (2011). Redes de Voluntariado de Adultos Mayores. Redes de Voluntariado de Adultos Mayores aportan al de- 
sarrollo, la democracia y al envejecimiento y activo y digno. Recuperado el 10 de marzo de 2020, de http://www. gestionsocial.org/ archivos/00000762/JBravo115.pdf.

Cussiánovich, A. (2007). Aprender la condición humana. Ensayo sobre pedagogía de la ternura. Lima: IFEJANT. Recuperado el 02 de marzo de 2020, de http://www. mnnatsop-peru.org/contenidos/index.php.

Cussiánovich, A. (2010). Ensayos sobre Infancia II. Sujeto de derechos y protagonista. Lima: IFEJANT.

Giró J. (Coord.) (2011). Envejecimiento activo, envejecimiento en positivo. Rioja: Universidad de Rioja Servicios de Publicaciones.

GonZÁLez, C. (2017). La intervención social en el campo gerontológico. Revista digital de Trabajo Social Conciencia Social. Vol. 1, Nro. 1 - ISSN [en trámite]. Universidad Nacional de Córdoba, Facultad de Ciencias Sociales. Recuperado el 12 de marzo de 2020, de https://revistas.unc.edu.ar/index.php/ConCienciaSocial/.

Golte, J. y León, D. (2011). Polifacéticos: jóvenes limeños del siglo XXI. Lima: Instituto de Estudios Peruanos.

Hartu-Emanak (2007). Las personas mayores en el umbral del siglo XXI. Ciudadania y Participación Social. Bilbao: Marra, España: Hartu-Emanak.

Hartu-Emanak (2009). Aprendizaje permanente. Participación social. Una perspectiva del envejecimiento activo desde Hartu-Emanak. Bilbao, Bizkaia/Vizcaya, España: Hartu-Emanak.

Instituto DE Estudios Latinoamericanos (s.f.). Participación social. Freie Universitat Berlin. Recuperado el 10 de abril de 2020, de https://www.lai. fu-berlin.de/es/e-learning/projekte/frauen_konzepte/ projektseiten/frauenbereich/rott/RO_Konzepte/RO_ participacion/index.html

Instituto de Mayores y Servicios Sociales (2008). Envejecimiento activo, libro blanco. Madrid: IMSERSO Publicaciones.

Instituto de Mayores y Servicios Sociales (2011). Participación social de las personas mayores. Madrid: IMSERSO Publicaciones.

Instituto Nacional de Estadística e Informática (2020). Situación de la población adulta mayor. Octubre - Noviembre - Diciembre 2019. Informe Técnico $N^{\circ}$ 1. Marzo 2020. Lima: INEI. Recuperado el 12 de marzo de 2020, de https://www.inei.gob.pe/media/ MenuRecursivo/boletines/boletin_adulto_mayor_ oct_nov_dic2019.pdf.
Iturrieta F. (2008). Participación Social y la nueva articulación entre Estado, Mercado y Sociedad Civil. Análisis del cambio en la relación entre Estado, Mercado y Sociedad Civil, y su relación en la conformación de iniciativas de Participación Social. Tesis de pregrado de la Universidad de Chile, Santiago de Chile, Chile.

Jaramillo E. (2005). Protagonismo de niños, niñas y adolescentes. Una experiencia latinoamericana: El caso Perú. Ponencia ofrecida en el Seminario Significado y Pertinencia del Protagonismo de Niños, Niñas y Adolescentes, organizado por la Universidad Centroamericana (UCA). Nicaragua, Managua.

Miralles I. (2011). Envejecimiento productivo. Las contribuciones de las personas mayores desde la cotidianidad. Núcleo Básico de Revistas Científicas Argentinas del CONICET. No 16, vol. XV, verano 2011, Santiago del Estero, Argentina.

PIÑA, M. (2013). Reflexiones epistemológicas en la investigación del maltrato societal. En María Teresa Abusleme L., Máximo Caballero A. (ed.), Maltrato de las personas mayores en Chile. Santiago de Chile: Ediciones Servicio Nacional del Adulto Mayor.

PIÑA, M. (2009). Trabajo social gerontológico: investigando y construyendo espacios de integración social para las personas mayores. Universidad de Costa Rica - Escuela de Trabajo Social. Boletín Electrónico Surá Nro. 151, febrero 2009. Recuperado el 28 de febrero de 2020, de http://www.ts.ucr.ac.cr/binarios/sura/sura-0151.pdf.

Quintanar, F. (2000). Ancianos en asilos y casa hogar de la ciudad de México. Ante el escenario de la tercera ola. México DF: Plaza y Valdés.

Remy, M. (1991). Los discursos sobre la violencia en los Andes. Algunas reflexiones a propósito del Chiaraje. En Henrique Urbano (comp.), Mirko Lauer (ed.), Poder y violencia en los Andes. Cusco: Centro de Estudios Regionales Andinos Bartolomé de las Casas.

SÁnchez, C. (2005). Gerontología social. Buenos Aires: Espacio Editorial.

Thurner, M. (2006). Republicanos andinos. Lima: CBC e IEP.

VARela, L. y otros (2004). Desarrollando respuestas integradas de sistemas de cuidados de salud para una población en rápido envejecimiento INTRA II. Lima: Ministerio de Salud e Instituto de Gerontología de la Universidad Peruana Cayetano Heredia. 